\title{
Subcutaneous Hormonal Therapy
}

National Cancer Institute

\section{Source}

National Cancer Institute. Subcutaneous Hormonal Therapy. NCI Thesaurus. Code C116543.

The administration of exogenous hormones via bolus injection into the tissue layer immediately deep to the dermis. 